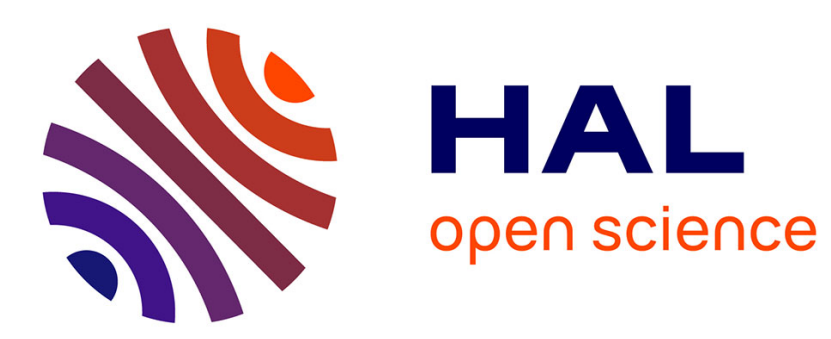

\title{
Optimizing Iterative Physical Optics by using an ACA compression on interaction matrices
}

Antoine Thomet, Gildas Kubicke, Christophe Bourlier, Philippe Pouliguen

\section{To cite this version:}

Antoine Thomet, Gildas Kubicke, Christophe Bourlier, Philippe Pouliguen. Optimizing Iterative Physical Optics by using an ACA compression on interaction matrices. 2015 IEEE International Symposium on Antennas and Propagation and North American Radio Science Meeting, Jul 2015, Vancouver, Canada. pp.\#3653, 10.1109/aps.2015.7305138 . hal-01120206

\section{HAL Id: hal-01120206 https://hal.science/hal-01120206}

Submitted on 11 May 2020

HAL is a multi-disciplinary open access archive for the deposit and dissemination of scientific research documents, whether they are published or not. The documents may come from teaching and research institutions in France or abroad, or from public or private research centers.
L'archive ouverte pluridisciplinaire HAL, est destinée au dépôt et à la diffusion de documents scientifiques de niveau recherche, publiés ou non, émanant des établissements d'enseignement et de recherche français ou étrangers, des laboratoires publics ou privés. 


\title{
Optimizing Iterative Physical Optics by using an ACA compression on interaction matrices
}

\author{
Antoine Thomet ${ }^{* \ddagger}$, Gildas Kubické ${ }^{\dagger}$, Christophe Bourlier ${ }^{*}$ and Philippe Pouliguen ${ }^{\ddagger}$ \\ *IETR (Institut d'Electronique et des Télécommunications de Rennes) Laboratory, CNRS UMR 6164 \\ LUNAM Université, Université de Nantes, La Chantrerie, 44306 Nantes, France \\ Email: antoine.thomet@univ-nantes.fr \\ ${ }^{\dagger}$ DGA Information Superiority \\ CGN1 division, 35170 Bruz, France \\ ${ }^{\ddagger}$ DGA/DS/MRIS (Direction Générale de l’Armement - Direction de la Stratégie - \\ Mission pour la Recherche et l'Innovation Scientifique) 92221 Bagneux, France
}

\begin{abstract}
This paper presents a numerical compression of Iterative Physical Optics (IPO), known as an efficient asymptotic method for computation of Radar Cross Section (RCS) of complex targets including cavities. The compression is applied to interaction matrices of IPO between coupled surfaces. It is made by using the algebraic Adaptive Cross Approximation (ACA), which is known to provide a good compression and acceleration when used with dense matrices involved in rigorous numerical methods. Results show that this method highly compresses the IPO matrices, without damaging the RCS prediction by IPO.
\end{abstract}

\section{INTRODUCTION}

Computing Radar Cross Section (RCS) of large targets including cavities (such as aircrafts inlets) is an important issue in radar detection. An efficient computational method is needed for such complex targets. In this context, two categories of numerical methods can be used.

Rigorous methods are known to provide a good accuracy, as they do not apply any approximation but approximation due to meshing. Nevertheless, their complexity is very high, so these methods are difficult to use for targets much greater than the wavelength, due to their computation time and memory requirements.

For electrically large targets, one can use High Frequency asymptotic methods, among which Iterative Physical Optics (IPO) [1], [2]. IPO is an asymptotic method based on Physical Optics (PO), which is known to provide a good compromise between complexity and precision [1], [2].

This paper presents an optimization of IPO by using a matrix compression method: Adaptive Cross Approximation (ACA) [3]. It is applied here to IPO matrices of coupled surfaces, splitted into 2 separated objects. The method enables to highly reduce the memory storage and the complexity.

\section{ITERATIVE PhysiCAL OPTICS APPLIED TO COUPLED OBJECTS}

\section{A. Method}

IPO is an asymptotic method based on PO, using surface currents to determine scattered fields. It is used to compute the scattering by complex targets (cavities, but also complex objects with interacting surfaces). The method can be described in a 3 steps algorithm:
1) Iteration 0: this first step gives the initial value of the surface curent. Several techniques can be used in order to determine these expressions [1];

2) Iterative process: surface currents induce fields scattering towards the structure. These fields imply an iterative update of surface currents, using PO approximation;

3) Scattering through the receiver: after convergence, currents are used to determine the field scattered by the structure.

\section{B. IPO Matrices for coupled objects}

Considering two separated objects, the update of the surface current (step 2 of the IPO algorithm) is made among the following equations:

$$
\begin{aligned}
& \bar{J}_{1}^{(k+1)}=\bar{J}_{1}^{(0)}+\overline{\mathbf{C}}_{11} \times \bar{J}_{1}^{(k)}+\overline{\mathbf{C}}_{21} \times \bar{J}_{2}^{(k)} \\
& \bar{J}_{2}^{(k+1)}=\bar{J}_{2}^{(0)}+\overline{\mathbf{C}}_{12} \times \bar{J}_{1}^{(k)}+\overline{\mathbf{C}}_{22} \times \bar{J}_{2}^{(k)}
\end{aligned}
$$

Where $\bar{J}_{1}^{(k)}$ and $\bar{J}_{2}^{(k)}$ are the current vectors of surfaces 1 and 2, respectively, at the iteration $k$, and $\overline{\mathbf{C}}_{a b},(a, b) \in\{1,2\}$, is the matrix representing the effect of current of surface $a$ on the update of current of surface $b$.

\section{Expressions in cartesian coordinates}

Expressions of $\bar{J}_{a}^{(k)}$ and $\overline{\mathbf{C}}_{a b},(a, b) \in\{1,2\}$, depend on the coordinates system. In cartesian $(X, Y, Z)$ coordinates, $\bar{J}_{a}^{(k)}$ is of size $\left[3 N_{a} \times 1\right]$, and $\overline{\mathbf{C}}_{a b}$ is of size $\left[3 N_{b} \times 3 N_{a}\right]$, where $N_{a}$ and $N_{b}$ are the number of elements of surfaces $a$ and $b$, respectively. For any $(a, b), \overline{\mathbf{C}}_{a b}$ is expressed from $9\left[N_{b} \times N_{a}\right]$ blocks:

$$
\overline{\mathbf{C}}_{a b}=\left[\begin{array}{lll}
\overline{\mathbf{C x x}} & \overline{\mathbf{C y x}} & \overline{\mathbf{C z x}} \\
\overline{\mathbf{C x y}} & \overline{\mathbf{C y y}} & \overline{\mathbf{C z y}} \\
\overline{\mathbf{C x z}} & \overline{\mathbf{C y z}} & \overline{\mathbf{C z z}}
\end{array}\right]
$$

In case of a Perfect Electric Conductor (PEC), the expressions are the following ones, where $(p, q, r) \in\{X, Y, Z\}$, and by separating the diagonal blocks from the other ones: 


$$
\begin{aligned}
\overline{\mathbf{C p p}}(u, v)= & {\left[n_{q}(v) \alpha P(u, v) \Delta S(u) k_{q}(u, v)\right.} \\
& \left.+n_{r}(v) \alpha P(u, v) \Delta S(u) k_{r}(u, v)\right] I_{c}(u, v) \\
\overline{\mathbf{C p q}}(u, v)= & -\left[n_{p}(v) \alpha P(u, v) \Delta S(u) k_{q}(u, v)\right] I_{c}(u, v)
\end{aligned}
$$

In these equations, $n_{p}(v)$ is the $p$ component of unitary normal vector of element $v$ of $S_{b} ; \alpha=\frac{-2 j Z_{0}}{4 \pi \omega_{0} \mu_{0}}, Z_{0}$ being the impedance in free space, $\omega_{0}$ the pulsation, and $\mu_{0}$ permeability in free space; $P(u, v)=\left(k^{2} r^{2}+j k r\right) \frac{\exp (j k r)}{r^{3}}$ depends on distance $r$ between the element $u$ of $S_{a}$ and the element $v$ of $S_{b} ; \Delta S(u)$ is the area of the element $u$ of $S_{a} ; k_{q}(u, v)$ is the $q$ component of unitary vector pointing from the element $u$ of $S_{a}$ to the element $v$ of $S_{b}$; and $I_{c}(u, v)$ is a boolean showing whether or not the element $u$ of $S_{a}$ illuminates the element $v$ of $S_{b}$ in the iterations.

\section{AdAPTIVE CROSS APPROXIMATION}

\section{A. The ACA compression}

The Adaptive Cross Approximation (ACA) [3] is a matrix compression method consisting in the transformation of a matrix into the product of two smaller ones:

$$
\overline{\mathbf{Z}}[M \times N] \simeq \overline{\mathbf{U}}[M \times R] \times \overline{\mathbf{V}}[R \times N]
$$

Where $R$ is called the effective rank of matrix $\overline{\mathbf{Z}}$, and $R \ll(M, N)$. The method is purely algebraic and based on an algorithm, described in [3], minimizing the error $\epsilon_{A C A}$ between $\overline{\mathbf{Z}}$ and the product $\overline{\mathbf{U}} \times \overline{\mathbf{V}}$.

For electromagnetics applications, to the authors knowledge, ACA has been used only with rigorous methods, applied to reduce the complexity of impedance matrix inversion [3]. It is known to provide excellent compression ratio.

\section{B. Applying ACA compression to IPO matrices}

In this paper, an application of ACA to interactions IPO matrices between two plane objects is proposed. For such a case, matrices $\overline{\mathbf{C}}_{11}$ of (1) and $\overline{\mathbf{C}}_{22}$ of (2) are null as PO does not consider creaping waves. Then ACA algorithm can be applied to $\overline{\mathbf{C}}_{12}$ and $\overline{\mathbf{C}}_{21}$, in order to reduce:

- the memory requirement. Matrices $\overline{\mathbf{C}}_{12}$ and $\overline{\mathbf{C}}_{21}$ both contain $N_{1} \times N_{2}$ elements to store, whereas storing matrices $\overline{\mathbf{U}}$ and $\overline{\mathbf{V}}$ from ACA compression reduces it to $\left(N_{1}+N_{2}\right) R$ elements, with $R \ll(M, N)$;

- the complexity and so the computation time for each iteration. The number of operations in the iterations (step 2 of IPO algorithm) is also reduced from $\mathcal{O}\left(N_{1} \times N_{2}\right)$ (for $\overline{\mathbf{C}} \times \bar{J}$ matrix-vector products) to $\mathcal{O}\left(\left(N_{1}+N_{2}\right) R\right)$ elements (for $\overline{\mathbf{U}} \times(\overline{\mathbf{V}} \times \bar{J})$ matrix-vector products), with $R \ll(M, N)$, this operation being repeated twice per iteration (applied to $\overline{\mathbf{C}}_{12}$ and $\overline{\mathbf{C}}_{21}$ ).

\section{NUMERICAL RESULT}

The result shown in Fig. 1 is the VV bistatic RCS of a thin right-angled dihedral. The dihedral is made of two square faces of length $8 \lambda$, the frequency being $10 \mathrm{GHz}$. The proposed IPO+ACA method is applied to compute the interactions between the two internal faces of the dihedral. The incidence direction is the dihedral's median and the observation varies by rotation around the internal edge, limited to the interior of the dihedral. The ACA threshold is $\epsilon_{A C A}=5 \cdot 10^{-5}$.

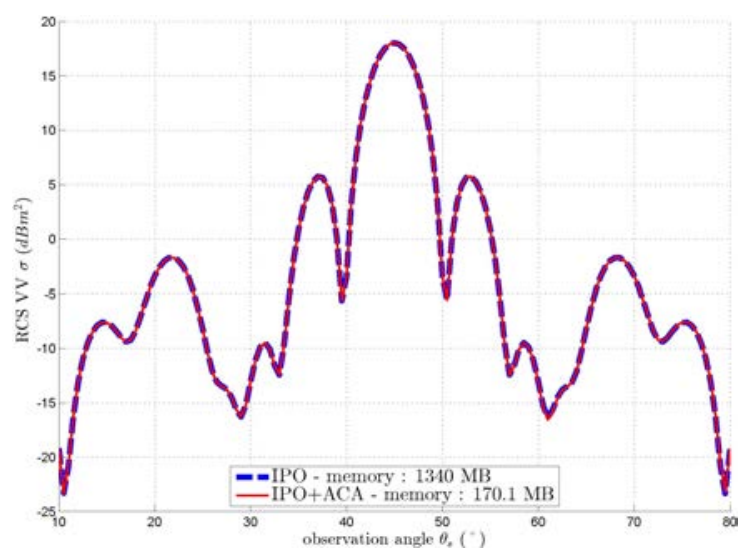

Fig. 1. Bistatic RCS of a thin PEC dihedral illuminated from its median

One observe in Fig. 1 that the result obtained by the IPO+ACA method is very close to the one obtained by classical IPO. The relative error between the two results is $0.49 \%$, while the memory requirements are highly reduced: the compression ratio obtained by using ACA is $87.3 \%$.

\section{CONCLUSION}

This paper presents a low computational cost method for calculation of RCS of complex targets. By using a numerical compression, the proposed method allows to reduce consequently the memory and computation requirements. The presented result shows the efficiency of the method, which will be generalized to more complex targets, including complete cavities.

\section{REFERENCES}

[1] A. Thomet, G. Kubické, C. Bourlier, and P. Pouliguen, "Improvement of iterative physical optics using the physical optics shadow radiation", Progress In Electromagnetics Research M, vol. 38, pp. 1-13, 2014.

[2] F. Obelleiro-Basteiro, J. L. Rodriguez, and R. J. Burkholder, "An Iterative Physical Optics approach for analyzing the electromagnetic scattering by large open-ended cavities", IEEE Trans. Antennas Propagat., vol. 43, no. 4, pp. 356-361, 1995.

[3] K. Zhao, M. N. Vouvakis, and J.-F. Lee, The Adaptive Cross Approximation algorithm for accelerated method of moments computations of EMC problems, IEEE Trans. on Electromagnetic Compatibility, vol. 47, no. 4, pp. 763-773, Nov 2005. 